\title{
Coded Modulation with APSK for OFDM-Based Visible Light Communications
}

\author{
Qi Wang ${ }^{1}$, Zhaocheng Wang ${ }^{1}$, and Jinguo Quan ${ }^{2}$ \\ ${ }^{1}$ Tsinghua National Laboratory for Information Science and Technology (TNList) \\ Department of Electronic Engineering, Tsinghua University, Beijing 100084, China \\ ${ }^{2}$ Division of Information Science \& Technology, Shenzhen Graduate School \\ Tsinghua University, Shenzhen 518055, China \\ qiwang11@mails.tsinghua.edu.cn
}

\begin{abstract}
Coded modulation with APSK is proposed for OFDM-based VLC systems. Simulation results show that the proposed scheme achieves better performance than conventional QAM counterpart in both DCO-OFDM and ACO-OFDM systems, while similar complexity is maintained.
\end{abstract}

OCIS codes: (060.2605) Free-space optical communication; (060.4080) Modulation.

\section{Introduction}

Motivated by the dramatic development of light emitting diode (LED) technologies as well as the scarce spectrum of the radio frequency (RF), visible light communication (VLC) has gained increasing attention and has considered as a promising complementary technique to RF counterpart [1]. Recently, orthogonal frequency division multiplexing (OFDM) is employed in VLC systems because of its inherent resistance to inter-symbol interference and high spectral efficiency, and up to gigabit per second transmissions have been reported [2]. A number of optical OFDM schemes have been proposed, among which DC-biased optical OFDM (DCO-OFDM) and asymmetrically clipped optical OFDM (ACO-OFDM) are commonly used [3]. In conventional OFDM-based VLC systems, the subcarriers are modulated by quadrature amplitude modulation (QAM). The bit error rate (BER) considered is as high as $2 \cdot 10^{-3}$ and forward error correction (FEC) code is not considered in the optimization of VLC systems [2,3]. Amplitude phase shift keying (APSK) with Gray mapping [4] is recently proposed, which provides considerable shaping gain since its distribution is more close to Gaussian compared with QAM. When FEC codes such as low-density parity-check (LDPC) code and Turbo code are utilized, APSK is expected to achieve better performance than QAM.

In this paper, coded modulation is investigated for VLC systems, where LDPC code is employed as FEC code and APSK constellation is adopted for modulation instead of traditional QAM. Low-complexity soft demapper for APSK is applied at the receiver, which has similar complexity as that of QAM. Simulation results show that the proposed approach significantly improves the BER performance of VLC systems. Besides that, the proposed coded modulation with APSK scheme outperforms its QAM counterpart in both DCO-OFDM and ACO-OFDM systems.

\section{Coded Modulation with APSK for OFDM-based VLC systems}

The block diagram of OFDM-based VLC system with coded modulation is shown in Fig. 1. At the transmitter, the information bits are encoded by the FEC encoder. The coded bits are then mapped from bits to symbols, where QAM or APSK constellation is employed. The modulated symbols are serial-to-parallel (S/P) converted and Hermitian symmetry is imposed to guarantee that the time-domain signals after inverse fast Fourier transform (IFFT) are realvalued. In DCO-OFDM, the bipolar time-domain signals are forced to be non-negative by a DC bias proportional to the power of time-domain signal. In ACO-OFDM, however, only the odd subcarriers are modulated, leading to an anti-symmetric waveform after IFFT, which can be directly clipped at zero without information loss. After DC biasing or clipping, the unipolar electrical signals are used to modulate the instantaneous power of LEDs and emitted into the air. At the receiver, the optical signal is detected by an avalanche photodiode (APD) and converted to the electronic signal. After S/P conversion, the received time-domain signals are transformed to frequency domain by FFT. Unlike the traditional systems where the hard decision is applied to the frequency-domain symbols, soft demapper is employed in the proposed system, which calculates the log-likelihood ratio (LLR) for each bit as the input of the FEC decoder.

An $M$-APSK constellation is composed of $R$ concentric rings, each with uniformly spaced PSK points. The $M$ APSK constellation set is given by $\mathscr{X}=\left\{r_{l} \exp \left(j\left(2 \pi i / n_{l}+\theta_{l}\right)\right) \mid i=0, \cdots, n_{l}-1,0 \leqslant l<R\right\}$. Product-APSK with 


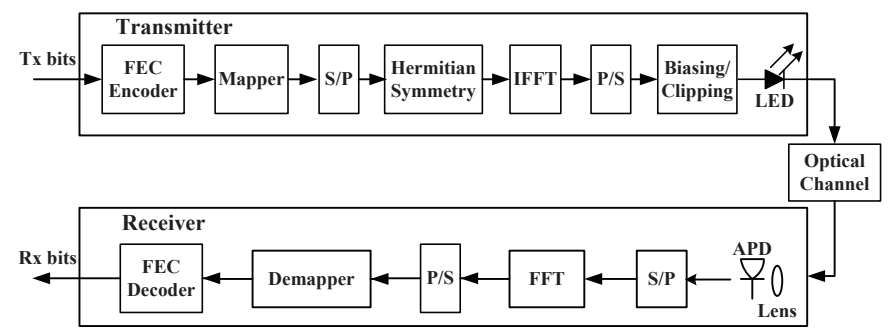

Fig. 1. Block diagram of OFDM-based VLC system with coded modulation.

Gray mapping [4] was proposed with $R=2^{m_{2}}$ rings and $n_{l}=2^{m_{1}}$ points on each ring for $M=2^{m}$-ary APSK, where we have $m_{1}+m_{2}=m$. The $2^{m}$-APSK can be decomposed into a $2^{m_{1}}$-ary PSK and a pseudo $2^{m_{2}}$-ary PAM. Compared with traditional QAM, the non-uniformly spaced APSK constellation is more close to Gaussian distribution, which can achieve considerable shaping gain and more average mutual information (AMI). Therefore, when FEC code is considered in coded modulation, APSK is expected to achieve better performance than QAM.

\section{Low-Complexity Soft Demapper for APSK}

For advanced FEC codes such as LDPC code and Turbo code, soft information for each bit is required for their corresponding decoders to achieve near Shannon limit performance, where soft demapper instead of hard decision is required to demodulate the symbols at the receiver. The widely used algorithm for soft demapper is Max-Log-MAP, and the soft information of the $i$-th bit is expressed in the form of LLR as [5]

$$
L_{i}=-\frac{1}{N_{0}}\left(\min _{X \in \mathscr{X}_{i}^{(0)}}|Y-H X|^{2}-\min _{X \in \mathscr{X}_{i}^{(1)}}|Y-H X|^{2}\right)
$$

for $0 \leq i<m$, where $X$ and $Y$ are the transmitted and received symbols in the frequency domain, $H$ denotes the frequency-domain channel impulse response, $N_{0}$ represents the variance of the noise, and $\mathscr{X}_{i}^{(b)}$ stands for the signal subset of $\mathscr{X}$ with the $i$-th bit being $b \in\{0,1\}$.

The Max-Log-MAP demapper need to calculate all the $2^{m}$ squared Euclidean distances, i.e. $|Y-H X|^{2}$ for every $X \in \mathscr{X}$, in order to find the two minimum terms described in Eq. (1). Therefore, its computational complexity is on the order of $\mathrm{O}\left(2^{m}\right)$ for $M=2^{m}$-ary constellations, which is unacceptable especially for VLC systems where high order modulations are commonly used to achieve high spectral efficiency.

In QAM constellations, the complexity can be directly reduced to the order of $\mathrm{O}\left(2^{m / 2}\right)$ by decomposing it into two $2^{m / 2}$-ary PAMs, which can be further reduced to the order of $\mathrm{O}(\mathrm{m})$ when a piecewise linear approximation is invoked [5]. Similar to QAM demapper, the complexity of APSK demapper can be reduced to the order of O $\left(2^{m_{1}}\right)+$ $\mathrm{O}\left(2^{m_{2}}\right)$ by decomposing APSK into $2^{m_{1}}$-ary PSK and a pseudo $2^{m_{2}}$-ary PAM. After FFT at the receiver, the complexvalued symbol $Y$ is firstly Cartesian-to-polar converted as $Y=\rho_{Y} \varphi_{Y}$, where $\rho_{Y}$ and $\varphi_{Y}$ denote the amplitude and phase of $Y$. After that, the LLRs of bits corresponding to amplitude and phase are calculated by the PAM and PSK demappers. By exploiting the symmetry of Gray-labeled constellations for PSK and PAM, the complexity is further reduced to $\mathrm{O}(m)$ [5], which is the same as QAM demapper. Therefore, the proposed coded modulation with APSK scheme has the similar complexity as its QAM counterpart, which can be directly applied to state-of-the-art VLC systems.

\section{Simulation Results}

The BER performance of the proposed system is evaluated via simulations in terms of the bit energy to noise power ratio $E_{b} / N_{0}$. In our simulations, the LDPC code in the IEEE 802.11 standard with a codeword-length of 648 bits and a code rate of $2 / 3$ is applied. Belief propagation (BP) algorithm is employed in the LDPC decoder and the maximum number of decoding iterations is set to 10 . The number of subcarriers is 512, and the useful subcarriers are modulated by Gray-QAM and Gray-APSK for comparison. Soft information for LDPC decoder is obtained by Max-Log-MAP algorithm, where LLR is calculated for each bit. Two different optical OFDM schemes are utilized, i.e., DCO-OFDM and ACO-OFDM. In DCO-OFDM, $13 \mathrm{~dB}$ DC bias is added to the time-domain signals to obtain unipolar signals [3].

The BER performance of DCO-OFDM-based VLC system is shown in Fig. 2(a). It can be seen that QAM outperforms APSK in uncoded systems since APSK constellation is nonuniform, which leads to more error probability when 


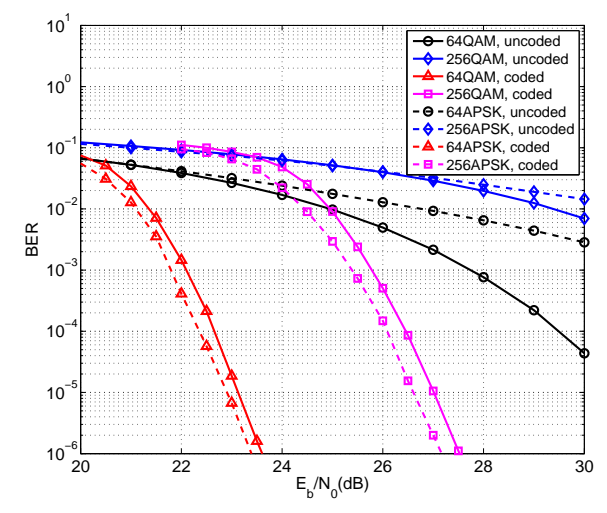

(a) DCO-OFDM-based VLC system with $13 \mathrm{~dB}$ DC bias

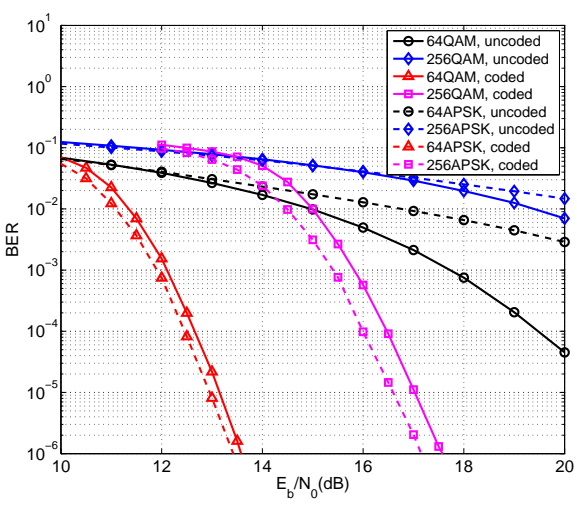

(b) ACO-OFDM-based VLC system

Fig. 2. BER performance of coded modulation with QAM and APSK.

hard decision is used. When LDPC code and soft demapper are adopted, the BER is reduced significantly. Moreover, unlike the uncoded systems, APSK achieves better performance than QAM in LDPC-coded modulation systems because of its shaping gain. Specifically, for 64-ary and 256-ary constellations, the performance gains of APSK over QAM are $0.22 \mathrm{~dB}$ and $0.36 \mathrm{~dB}$ at the BER of $10^{-6}$, respectively.

The BER performance of proposed scheme is also verified in ACO-OFDM-based VLC system, and the simulation results are shown in Fig. 2(b). It can be seen that ACO-OFDM achieves considerable performance gain compared with DCO-OFDM since DC bias is not required. Similar to the results of DCO-OFDM systems, the proposed coded modulation with APSK scheme still outperforms its QAM counterpart, and $0.16 \mathrm{~dB}$ and $0.42 \mathrm{~dB}$ gains are achieved at the BER of $10^{-6}$ in 64-ary and 256-ary scenarios. Therefore, coded modulation with APSK constellation is a better candidate for OFDM-based VLC systems.

\section{Conclusion}

In this paper, coded modulation with APSK is proposed for OFDM-based VLC systems, where LDPC code is employed as FEC code and APSK instead of QAM is adopted for modulation. Meanwhile, low-complexly soft demapper for APSK is applied at the receiver, which has similar complexity as that of QAM. Simulation results show that the BER performance is improved significantly when FEC code is employed, and the proposed coded modulation with APSK scheme achieves better performance than its QAM counterpart in both DCO-OFDM and ACO-OFDM systems.

\section{Acknowledgment}

This work was supported by National Key Basic Research Program of China (Grant No. 2013CB329203), National Nature Science Foundation of China (Grant No. 61271266), Shenzhen Visible Light Communication System Key Laboratory (ZDSYS20140512114229398) and Shenzhen Peacock Plan (No. 1108170036003286).

\section{References}

1. A. Jovicic, J. Li, and T. Richardson, "Visible light communication: opportunities, challenges and the path to market," IEEE Commun. Magazine, vol. 51, no. 12, pp. 26-32, Dec. 2013.

2. G. Cossu, A. M. Khalid, P. Choudhury, R. Corsini, and E. Ciaramella, "3.4 Gbit/s visible optical wireless transmission based on RGB LED,” Opt. Exp., vol. 20, no. 26, pp. B501-B506, Dec. 2012.

3. S. D. Dissanayake and J. Armstrong, "Comparison of ACO-OFDM, DCO-OFDM and ADO-OFDM in IM/DD systems," J. Lightwave Technol., vol. 31, no. 7, pp. 1063-1072, Apr. 2013.

4. Z. Liu, Q. Xie, K. Peng, and Z. Yang, "APSK constellation with Gray mapping," IEEE Commun. Lett., vol. 15, no. 12, pp. 1271-1273, Dec. 2011.

5. Q. Wang, Q. Xie, Z. Wang, S. Chen, and L. Hanzo, "A universal low-complexity symbol-to-bit soft demapper," IEEE Trans. Veh. Technol., vol. 63, no. 1, pp. 119-130, Jan. 2014. 